\title{
A case of COVID - 19 with Imported Falciparum Malaria Infection is Reported
}

\section{Mingchao Zhu ( $D$ 645249756@qq.com )}

the First people's Hospital of tianmen city

\section{Ya Zhu}

the first people's hospital of tianmen

Jue Zhang

the first people's hospital of tianmen

\section{Weiping Liu}

the first people's hospital of zigong

\section{Case report}

Keywords: Imported,P. falciparum,COVID - 19,SARS-CoV-2

Posted Date: June 9th, 2020

DOI: https://doi.org/10.21203/rs.3.rs-32935/v1

License: (9) This work is licensed under a Creative Commons Attribution 4.0 International License. Read Full License 


\section{Abstract}

Background During the COVID - 19 outbreak, limited medical resources in the short term and inadequate experience in dealing with major new public health events may lead to the neglect of some other infectious diseases, such as malaria, leading to the risk of the spread of infectious diseases.Therefore, it is particularly important to formulate classified guidance, take scientific prevention and control measures in a comprehensive manner, strengthen the screening of malaria patients and provide access to medical treatment during the outbreak.

Methods Clinical records, laboratory results, and chest CT scans were retrospectively reviewed for a falciparum malaria infection patient with laboratory-confirmed COVID - 19 pneumonia .

Results Patient's serum novel coronavirus antibody tested positive for IgG and weakly positive for IgM that were positive for severe acute respiratory syndrome coronavirus 2 [SARS-CoV-2],the blood samples were classified as plasmodium falciparum by RT-PCR.

Conclusions The patient when workers infected with $\mathrm{p}$. falciparum in Africa, malaria recurrence after back to China, after antimalarial treatment in tianmen city first people's hospital, after an outbreak in wuhan will be coronavirus, and symptoms of COVID - 19, but soon be cured patients, to explore the diagnosis and treatment of antimalarial drugs in COVID - 19 patients with the role, for further in-depth study of COVID - 19 treatment provides a good example.

\section{Introduction}

In December 2019, an outbreak of COVID - 19 broke out in wuhan, China, and quickly spread to the whole country. Currently, The Chinese government as positive such as sealing city, closing schools and work places of social isolation, such as public health interventions to make COVID - 19 outbreak in China has been effectively controlled [1], but imported cases continue to appear. There were 1713 confirmed cases imported from abroad [2]. Meanwhile,global outbreak is still ongoing, resulting in other diseases such as malaria and other infectious diseases being neglected due to the impact of the epidemic.A case of imported falciparum malaria was reported and analyzed in China during the epidemic period to provide a reference for the prevention and control of imported malaria during the epidemic period of COVID -19 .

Malaria is also a class B infectious disease that one of the most health threatening diseases in the world. It once caused a world pandemic and is an important public health issue of global concern. In recent years, China has made remarkable achievements in anti-malaria measures. But as a result of labor export, tourism, business, such as frequent population movements, there is an upward trend in overseas imported malaria cases, and in the majority with falciparum [4],Every year,there were nearly 3,000 imported malaria cases in China, imported falciparum is a serious threat to people's health [5] [6] [7], For these patients, if the diagnosis and treatment of cases and the investigation and treatment of epidemic sites cannot be carried out in time,can lead to death at any time, It may also cause secondary transmission and threaten our efforts to eliminate malaria [8]. Therefore, it is particularly important to 
strengthen the investigation of imported malaria and other infectious diseases during the COVID - 19 outbreak.

\section{Research In Context}

We retrospectively reviewed clinical records, laboratory findings,Morphological characteristics of the pathogen under microscope and chest CT scans for patent with laboratory-confirmed Plasmodium falciparum infection with COVID - 19 pneumonia. Evidence of Plasmodium falciparum infection with COVID - 19 was assessed by testing for the presence of SARS-CoV-2 in blood,throat swab sampales, plasmodium parasite and its nucleic acid in microscopic examination and blood sampales. The blood samples of the patients were confirmed as plasmodium falciparum by RDT or a mixture of three plasmodium parasites,Under the microscope, plasmodium trophozoite and PCR typing result were plasmodium falciparum.

\section{CASE PRESENTATION}

The patient is a 65-year-old man from Liuwan Village, Qianyi Town, Tianmen City, Hubei Province. On the afternoon of December 27, 2019, he was diagnosed with fever due to unknown causes. The outpatient was admitted to the hospital with "upper respiratory infection and fever, fever to be checked". The highest temperature was $39^{\circ} \mathrm{C}$ on admission., with headache, fatigue and other symptoms, physical examination: temperature $(\mathrm{T}): 38.1^{\circ} \mathrm{C}$, respiration (R): 20 beats / min, heart rate $(\mathrm{P}): 88$ beats / min, blood pressure (B): $130 / 80 \mathrm{mmHg}$. The patient was pale, with a lack of energy, with a clearer mind, with a regular heart rhythm, clear breathing in both lungs, no wet and dry rales, no symptoms of nausea, vomiting, abdominal pain and diarrhea. Physical examination revealed an old surgical scar about $8 \mathrm{~cm}$ long on the abdomen, soft abdomen, no tenderness and rebound pain,the underside of the liver could not be touch, normal bowel sounds, no edema in both lower limbs. Past medical history: The patient suffered from splenic rupture due to a fall and had a history of splenectomy in 2005. He has infected malaria while working in Africa in November 2019,. treated with antimalarial drugs, specific drugs and insect species unknown,,with no history of hypertension, diabetes, heart diseasenhepatitis, tuberculosis or food and drug allergy history .

The first blood cell analysis was performed on December 27, 2019: white blood cell count (WBC) $4.36 \times$ 109 cells / L[normal, 3.5-9.5 × 109 cells/L], red blood cell count (RBC) $3.81 \times 1012$ / L[normal, 4.3-5.8 $\times$ 1012 cells/L], hemoglobin (HGB) $124 \mathrm{~g} /$ L[normal, 130-175 g / L], thrombocytopenia (PLT) $77 \times 109$ cells / L[normal, 125-350 × 109 cells/L],

absolute neutrophil (NEU \#) 3.68 × 109 / L[normal, 1.8-6.3 × 109 cells/L], neutrophil ratio (NEU\%) 84.4\% [normal,40-75\%], and the C-reactive protein was $53.0 \mathrm{mg} / \mathrm{L}$ [normal, $\leq 6.0 \mathrm{mg} / \mathrm{L}$. Electrolyte: potassium ion $3.43 \mathrm{mmol} / \mathrm{L}[\mathrm{normal}, 3.5-5.3 \mathrm{mmol} / \mathrm{L}]$, sodium ion $132.7 \mathrm{mmol} / \mathrm{L}[\mathrm{normal}, 137-$ $147 \mathrm{mmol} / \mathrm{L}$, total calcium $2.02 \mathrm{mmol} / \mathrm{L}[\mathrm{normal}, 2.11-2.52 \mathrm{mmol} / \mathrm{L}]$, total carbon dioxide $21.8 \mathrm{mmol}$ / L[normal, 24-32 mmol / L], serum iron 2.4umol / L[normal, 10.6-36.7 mmol / L], magnesium ion 
$0.6 \mathrm{mmol} / \mathrm{L}[$ normal, 0.75-1.02 mmol / L], Inorganic phosphorus $0.83 \mathrm{mmol} / \mathrm{L}[$ normal, 0.85-1.51 mmol / L],liver function:total protein $51.15 \mathrm{~g} /$ L[normal, 65-85 g / L], albumin $28.5 \mathrm{~g} / \mathrm{L}[$ normal, $40-55 \mathrm{~g} / \mathrm{L}]$, and the rest were not significantly abnormal. Influenza virus antigen test: negative. Hemorrhagic fever virus antibody (IgG + IgM) test: negative. Gold labeling rapid malaria diagnostic reagent (RDT) test: It indicates that Plasmodium falciparum is positive, but it does not rule out simultaneous infection with the other three malaria parasites (Plasmodium malaria, oval malaria, and vivax malaria). Plasmodium microscopy: find a large number of Plasmodium ring bodies, thick blood plate counts, the density is about $6,872 / \mathrm{ul}$, the plasmodium parasite is small,the ring is slender, accounting for about $1 / 5$ of the entire red blood cell diameter, and the nucleus is tight, fuchsia, one or two nuclei, usually two can be seen on the ring of a Plasmodium(Fig. 1A); the cytoplasm is blue, the amount is small, trophozoite is often close to the edge of the cell, and the parasitic red blood cells do not swell,some even shrink, two or more trophozoite can be parasitic in a red blood cell (Fig. 1B); the trophozoite on the thick blood film are comma-shaped, exclamation marks, nucleus and cytoplasm are smaller, there are more trophozoite, the phenomenon of "Starry sky" was often presented(see Fig. 2C,D). The plasmodium parasite was immediately reported to the public health department of the hospital and the tianmen center for disease control and prevention according to the procedures for reporting infectious diseases. the CDC immediately dispatched medical staff from the department of infectious diseases to send the antimalarial drugs (artemether and piperaquine phosphate) to the hospital. The patient received dihydroartemisinin piperaquine tablets orally. 2 hours after taking the drug, the patient's fever was relieved

The patient reviewed the blood test December 28,2019: RBC $3.91 \times 1012$ / L, HGB $130 \mathrm{~g} / \mathrm{L}, \mathrm{PLT} 81 \times 109$ / L,CRP $31.73 \mathrm{mg} / \mathrm{L}$, patients with mild anemia, platelet reduction, but CRP decreased. Continue to observe the medication, and check the blood test again on December 30: RBC 3.46 $\times 1012$ / L, HGB $113 \mathrm{~g}$ / L, PLT $79 \times 109$ / L, NEU \# $1.80 \times 109$ / L, NEU\% 36.8\%, Absolute value of monocytes (MON \#) $0.80 \times$ 109 / L[normal, 0.1-0.6 109 cells/L], monocyte ratio (MON\%) 16.1\%[normal, 3-8\%], EOS \# $0.80 \times 109$ / L[normal, 0.02-0.52 109 cells/L], EOS\% 8.70\%[normal, 0.4-8\%], CRP $8.63 \mathrm{mg} / \mathrm{L}$, patient eosinophilic Both the absolute value and proportion of granulocytes increased, anemia further worsened, but platelets rebounded, CRP gradually returned to normal, indicating that the condition improved. Patients were treated with dihydroartemisinin and piperazine tablets, with a total dose of 8 tablets (each containing $40 \mathrm{mg}$ of dihydroartemisinin and $0.32 \mathrm{~g}$ of piperazine phosphate), 2 tablets / time, 4 tablets / day, 2 days as a course of treatment. After the end of a course of treatment, the fever symptoms have completely disappeared. Upon re-examination of the Plasmodium, a large number of Plasmodium rings are still visible on the microscope, but the cytoplasm has disappeared, leaving only purple naked nuclei, as shown in Fig. 3E[F. Dual-nuclear naked nuclei can be seen in some red blood cells., Or multiple naked cores, see Fig. 3B, C. Continue to consolidate the treatment and re-examine again after a course of treatment. The patient was negative for Plasmodium. The patient was asked to leave the hospital. The patient was instructed to review the Plasmodium 3 days later. No Plasmodium was found in the peripheral blood.

COVID - 19 after the outbreak of disease, the patient was identified as close contacts, was the emergency isolation under medical observation on January 23,2020 , during the patient had symptoms of fever, but 
negative for pharyngeal swab virus nucleic acid detection, 2019 - nCoV IgM antibody detection:weakly positive, IgG positive, lung CT imaging examination showed high density shadow grinding of glass samples, as shown in Fig. 4, microscopic examination of the malaria parasite negative, after the designated hospital for treatment,after 1 week of treatment, lung shadow gradually disappear, instruct patient to remain in isolation for 14 days, not again appear the symptom such as fever, and continue to follow-up to track the patients condition.

\section{Discussion}

Malaria symptoms caused by plasmodium falciparum often do not have the typical periodic high fever and chills, the symptoms change rapidly and the disease is severe. Severe falciparum malaria is as high as about $10 \%$ and the case fatality rate is close to $1 \%$ [9]. Plasmodium falciparum infection is the ability of red blood cells (IEs) through effective adhesion on vascular receptor on the host, it is a vicious and other major regional difference of the malaria parasite to infect humans, but also an important reason of p. falciparum particularly dangerous [10], confirmed for a long time, is also a important reason of falciparum malaria patients died, according to the report [11], imported falciparum malaria patients confirmed the average number of days for $(5.9 \pm 8.6) \mathrm{d}$, only $21.4 \%$ of diagnosed within $24 \mathrm{~h}$, the elders can reach $60 \mathrm{~d}$, easy to delay treatment, led to the deaths of patients, and is likely to cause secondary spread.

This patient was a migrant worker returning home in Angola, Angola, Africa. The epidemiological followup survey showed that the patient had malaria incidence two weeks (November) before returning to China, and returned to China after the symptoms of local treatment in Africa disappeared. On December 22,2019 symptoms such as fever and headache occurred. As a common cold treatment, after 5 days of treatment with oral cold medicine. The specific drug was unknown and ineffective. He was admitted to Tianmen First People's Hospital on December 27. The outpatient doctor issued the flu. Antigen and Plasmodium microscopic examination, rapid diagnosis of Plasmodium RDT-positive, suggested falciparum malaria. microscopic examination showed Plasmodium ring body, immediately treated with dihydroartemisinin piperaquine tablets, blood samples for PCR typing were sent to Center for Disease Control and Prevention of Hubei Province. The final result was P. falciparum. During the onset of the patient, a series of regular changes in blood routine occurred: PLT decreased during onset, neutrophils and CRP increased, RBC,HGB, PLT and CRP decreased on the second day of onset,and Anemia and PLT reduction continued to exist after a course of treatment, CRP gradually returned to normal, eosinophils began to increase, and mononuclearity increased. Eosinophilia is common in parasitic infections, which is closely related to type $\nabla$ hypersensitivity, and malaria is type $\nabla$ and type $\otimes$ hypersensitivity. In this case, the eosinophils did not increase in the early stage of malaria. The analysis may be caused by During the onset of malaria, the body is in an emergency state, and the secretion of adrenal cortex hormones increases, leading to a decrease in eosinophils [11]. In the later stage, the patient's fever is relieved and the eosinophil response increases. At the same time, protozoan metabolites, corpuscle proteins and red blood cell fragments enter the bloodstream and are engulfed by monocytes, causing an increase in monocytes. The patient's anemia gradually worsens due to Malaria mainly causes anemia through 
hemolysis and destruction of red blood cells, in addition, long-term subclinical malaria infection leads to inflammatory anemia [12].

After 2 days of treatment, although the antibody to the malaria parasite is still positive, microscopic examination revealed a major change in the morphology of the parasite. The plasmodium in the red blood cells has only naked nuclei and the cytoplasm has disappeared. Is this parasite no longer capable of dividing and proliferating, or It may be a dead worm, but further research is needed to confirm it. The patient had an outbreak of malaria in November. Without formal treatment, he returned to China in December (there is almost no local malaria in China, and the probability of reinfection is low). Then the malaria attack recurred. According to the definition of re-ignition (acute malaria patients Malaria treatment is incomplete, or because the body produces immunity, it eliminates most of the erythrocytes and stops the malaria parasites. Under certain conditions, a large number of malaria parasites remaining in the red blood cells proliferate in large numbers. After several weeks or months, In the case of infection, recurrence of seizures is called re-ignition). Considering the possibility of re-ignition of malaria, malaria reignition is often related to low host resistance and specific immunity and antigenic mutations of Plasmodium [13]. This patient is an elderly patient. There is no underlying disease, but there is a previous history of malaria. The liver function of the patient shows a significant decrease in total protein and albumin, and there are risk factors for low immunity. Coupled with electrolyte disorders and previous antimalarial anti-malarial treatment, it is inferred whether there is a malaria parasite antigen. Variation is possible, so people with malaria re-ignition should take regular, adequate doses and courses of antimalarial treatment.

At the same time, Plasmodium infection can also cause an overwhelming immune response in some people, which in turn releases a large number of metabolites and inflammatory mediators, resulting in damage to vascular endothelial cells and the formation of microthrombus, leading to extensive microcirculation disorders [14], The study also showed that when plasmodia-infected red blood cells (IEs) attach to tissue-bound receptors, they are called immobilized. When they attach to uninfected red blood cells, they roll off. In addition, endothelial inflammation is a feature of falciparum malaria, which may be directly caused by IEs attached to the endothelium, or indirectly caused by inflammatory host and parasite products (IE membrane component, hrp-2, etc.) [15][16]. which may be The main reason for the increase of D-dimer in patients. However, as the malaria parasite is cleared from the patient, the immune response returns to normal, and the microthrombus can be eliminated on its own.

Cured patients after discharge had symptoms of fever again, has a history of wuhan area contact, and CT have COVID - 19 performance, positive serological 2019 - nCoV IgG antibody detection and weakly positive of IgM, Previous infection or serological false positive findings were suggested, combining clinical symptoms and imaging examination report as COVID - 19 patients with clinical diagnosis cases, patients will be coronavirus (SARS - CoV - 2) negative nucleic acid detection, analysis may be due to the following reasons: on the one hand may be due to the limitations of sampling and testing methods of false negatives; On the other hand in will be joined the chloroquine phosphate as drugs for the treatment COVID - 19 of Novel Coronavirus (2019-nCoV) Infection by the National Health Commission (Trial Version 
7)[17], may also with the patient before resistance to malaria treatment (piperaquine phosphate and artemisinin) inhibits replication of the virus in the body, remove the body of SARS - CoV - 2 viruses, reduce viral load and lower than the detection limit, but the specific reason details still needs further research. According to the patient's contact history (he went to Wuhan railway station on January 18, 2020), and developed fever on January 23, presumably the patient was in the incubation period,which was consistent with the characteristics of COVID - 19 [18].

According to the comprehensive analysis, the patient was COVID - 19 with malaria attack. Most imported malaria cases returned home after a long journey, and the possibility of exposure to COVID - 19 infection was not excluded during the trip [20]. In this situation, screening for COVID - 19 should also be intensified to avoid missed diagnosis due to the overlong incubation period of novel coronavirus. When faced with the outbreak of major public health events, we should formulate classified guidance and scientific prevention and control measures that take into consideration [21],provide access for malaria patients. Meanwhile, we should strengthen the investigation of malaria and other imported diseases from abroad, so as to prevent the missed diagnosis and spread of other infectious diseases.

Both new coronavirus pneumonia and malaria are common challenges facing all humans [22]. The results and experience of China's elimination of malaria have been recognized by the international community. At present, China's experience in the prevention and control of COVID - 19 also provides a good reference for the prevention and control of the world epidemic.We call on all countries to join hands and learn from each other's experience to jointly win the battle against the global epidemic.

\section{Declarations}

\section{Conflict of Interest Statement}

All the authors declare no competing interests.

\section{Ethics approval}

This study was conducted with the patient's consent.

\section{Consent to participate}

Informed consent for participate was obtained from all participants.

\section{Funding}

This work was supported by the [Sichuan Medical Scientific Research Foundation] under Grant [Q18025]

\section{Availability of data and material}

The data and material used to support the findings of this study are available from the corresponding author upon request. 


\section{Author Contributions}

Mingchao Zhu and Ya Zhu conceived the study and they contributed equally to this manuscript,and supervised the research study; Jue zhang responsible for the analysis and provision of imaging and CT test results; Wei-ping Liu provided edited the manuscript; and all the authors have contributed to writing the manuscript.

\section{Acknowledgments}

We thank the patient in the study.

\section{References}

1. Zhang J. Litvinova M, Wang W, et al Evolving epidemiology and transmission dynamics of coronavirus disease 2019 outside Hubei province, China: a descriptive and modelling study [published online ahead of print, 2020 Apr 2. Lancet Infect Dis. 2020;S1473-3099(20)30230-9. dor:10.1016/S1473-3099(20)30230-9.

2. National Health and Wellness Commission. Update on the epidemic situation of new coronavirus pneumonia as of 24:00 on May 23 [EB / OL]. (2020-5-24). http://www.nhc.gov.cn/xcs/yqfkdt/202005/4b53986676de4619b71ddf160c2cd291.shtml.

3. Zhou Shuisen. Analysis of the progress and epidemic characteristics of malaria elimination nationwide in 2017 [J]. Chinese Journal of Parasitology Parasitic Diseases. 2018;36(03):201-9. Zhang Li F, Jun Z, Shaosen X Zhigui.

4. Tang Linhua. Diagnosis, treatment and management of imported malaria [M]. Shanghai: Shanghai Science and Technology Press; 2010. 11-3; 30-32.

5. MASCARELLO M. GOBBI F, ANGHEBEN A, et al. Imported malaria in immigrants to Italy: a changing pattern observed in north eastern Italy.[J]. J Travel Med,2009,5(5):317-21.

6. GONZALEZ A. NICOLAS JM, MUNOZ J. Severe imported malaria in adults: retrospective study of 20 cases.[J]. The American Journal of Tropical Medicine and Hygiene,2009,4(4):595-599.

7. D'ORTENZIO E, SISSOKO D, DEHECQ, JS, et al. Malaria imported into Reunion Island: is there a risk of re-emergence of the disease? [J]. Transactions of the Royal Society of Tropical Medicine and Hygiene,2010,4(4):251-254.

8. $10.16250 / \mathrm{j} .32 .1374 .2020036$

Zhu Guoding C Jun. Challenges of New Coronavirus Pneumonia Epidemic to China's Malaria Elimination and Countermeasures [J / OL]. Chinese Journal of Schistosomiasis Prevention and Treatment: 1-3 [2020-03-16]. doi.org/10.16250/j.32.1374.2020036.

9. Malaria Surveillance - United States

10.15585/mmwr.ss6612a1

Mace KE. Arguin PM. Malaria Surveillance - United States, 2014. MMWR Surveill Summ. 2017;66(12):1-24. Published 2017 May 26. doi:10.15585/mmwr.ss6612a1. 
10. Jensen AR. Adams Y, Hviid L. Cerebral Plasmodium falciparum malaria: The role of PfEMP1 in its pathogenesis and immunity, and PfEMP1-based vaccines to prevent it. Immunol Rev. 2020;293(1):230-52. doi:10.1111/imr.12807.

11. Zhu, Mingchao. Zhu Ya. A case report of imported oval malaria in Tianmen City [J]. Chinese Journal of Zoonoses. 2018;34(6):579-80.

12. Gasim G, Adam I. 2016. Malaria, Schistosomiasis, and Related Anemia. Available from:.http://cdn.intechopen.com/pdfs-wm/50656.pdf.

13. Zhu, Xinping, editor in chief, Su Chuan. Human parasitology [M]. Beijing. People's Medical Publishing House, 2016: 56-61.

14. Wang Y. Pathogenesis of malaria and its prevention and treatment [J]. International Journal of Epidemiology Infectious Disease. 2014;41(5):336-41. Feng Guohe.

15. Viebig NK. Wulbrand U, Forster R. Andrews KT, Lanzer M. Knolle PA. Direct activation of human endothelial cells by Plasmodium fal-ciparum-infected erythrocytes. Infect Immun. 2005;73:32713277.

16. Jambou R. Combes V, Jambou MJ. Weksler BB, Couraud PO. Grau GE. Plasmodium falciparum adhesion on human brain microvascular endothelial cells involves transmigration-like cup formation and induces opening of intercellular junctions. PLoS Pathog. 2010;6(7):e1001021. Published 2010 Jul 29. doi:10.1371/journal.ppat.1001021.

17. National Health and Health Commission. New Coronavirus Pneumonia Diagnosis and Treatment Scheme (Trial Version 7)

http://www.nhc.gov.cn/yzygj/s7653p/202003/46c9294a7dfe4cef80dc7f5912eb1989.shtml.

18. Huang C. Wang Y, Li X, et al Clinical features of patients infected with 2019 novel coronavirus in wuhan, China. Lancet, 2020, doi: 10.1016/S0140-6736(20)30183-5.

19. Cao Jun Z, Shuisen Z Huayun, et al. From Malaria to Elimination in China-Target Strategies and Measures in Elimination Stage [J]. Chinese Journal of Schistosomiasis Control, 2013, 25 (5): 43943.

20. Rodríguez Morales AJ. MacGregor K, Kanagarajah S, et al. Going global Travel and the 2019 novel coronavirus [J]. Travel Med Infect Dis, 2020, in press..

21. Kai Wu. Wan Zheng. Analysis of a case of imported malarial malaria during epidemic of new coronary pneumonia in Wuhan [J]China Tropical Medicine, 2020,3 (20): 203-5.

22. Liu Yaobao C. Jun. [Expert Perspective] The Enlightenment of China's Overseas Imported Malaria Prevention and Control Strategies on the Current New Coronavirus Pneumonia Prevention and Control Work. Chinese Journal of Schistosomiasis Prevention and Control, Schistosomiasis Editorial Department [2020-03-15].https : //mp.weixin.qq.com/s/uNd5im7yt_V3jGCBKCR9Hg.

\section{Figures}




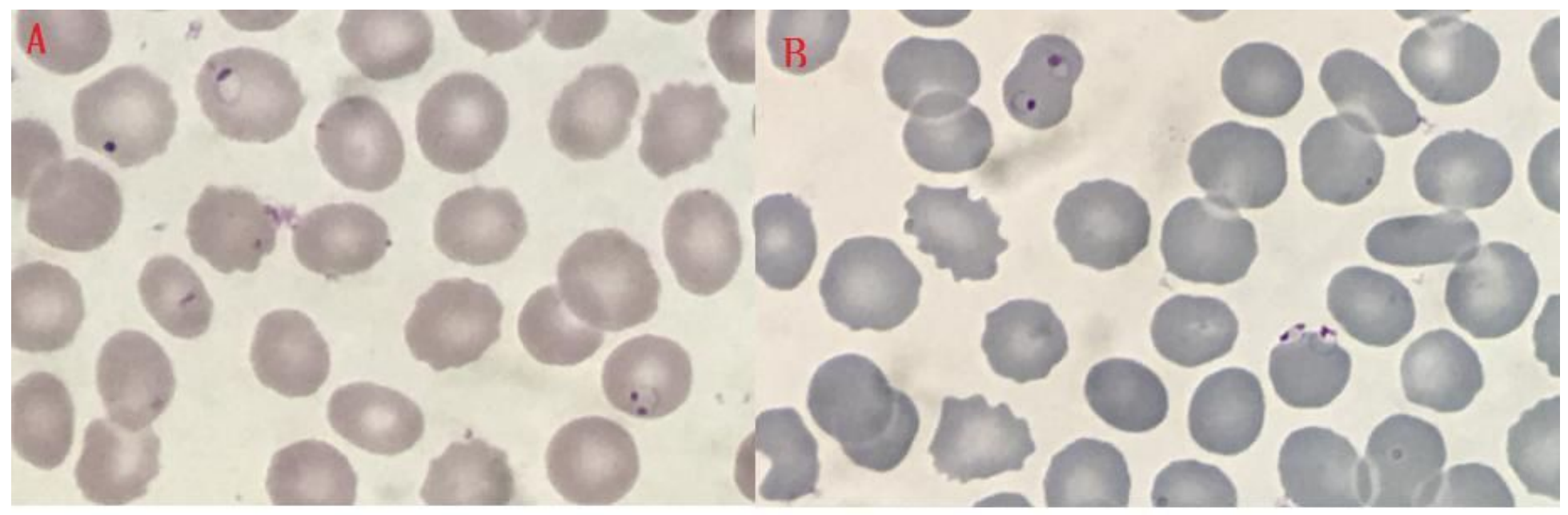

\section{Figure 1}

Plasmodium falciparum trophozoite(A囚B囚wright stain,thin blood film $\times 1,000)$

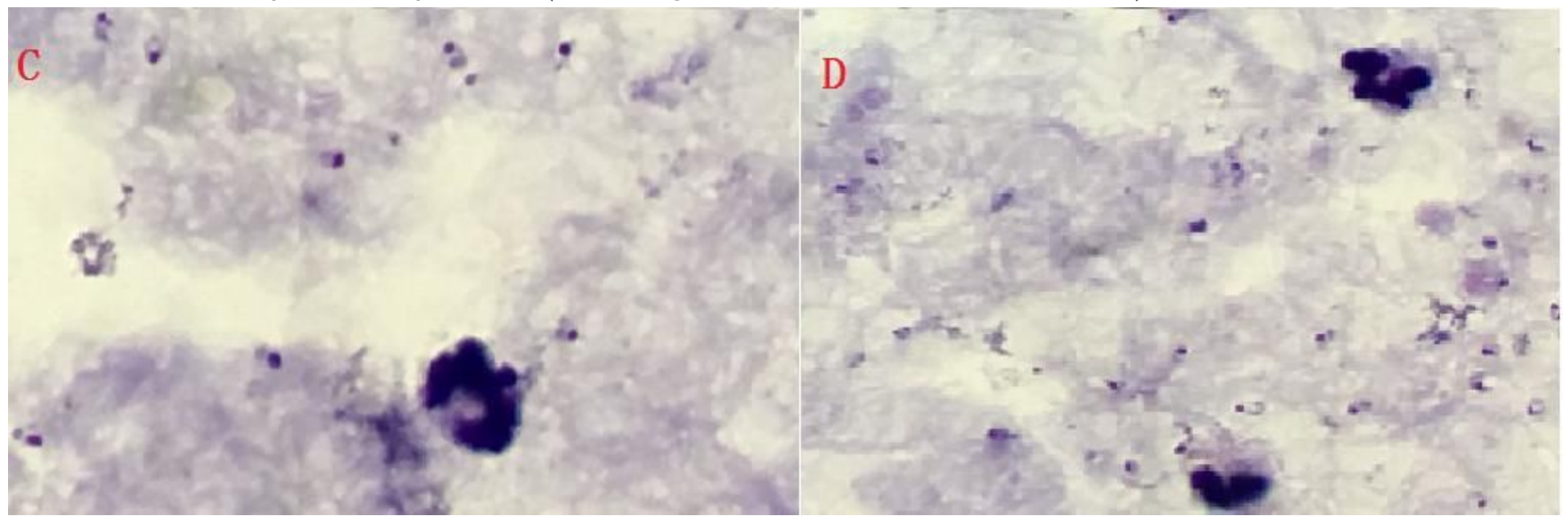

Figure 2

Plasmodium falciparum ring form ( $\mathbb{D} \mathbb{D} \llbracket$ Giemsa stain, thick blood film $\times 1,000$ )

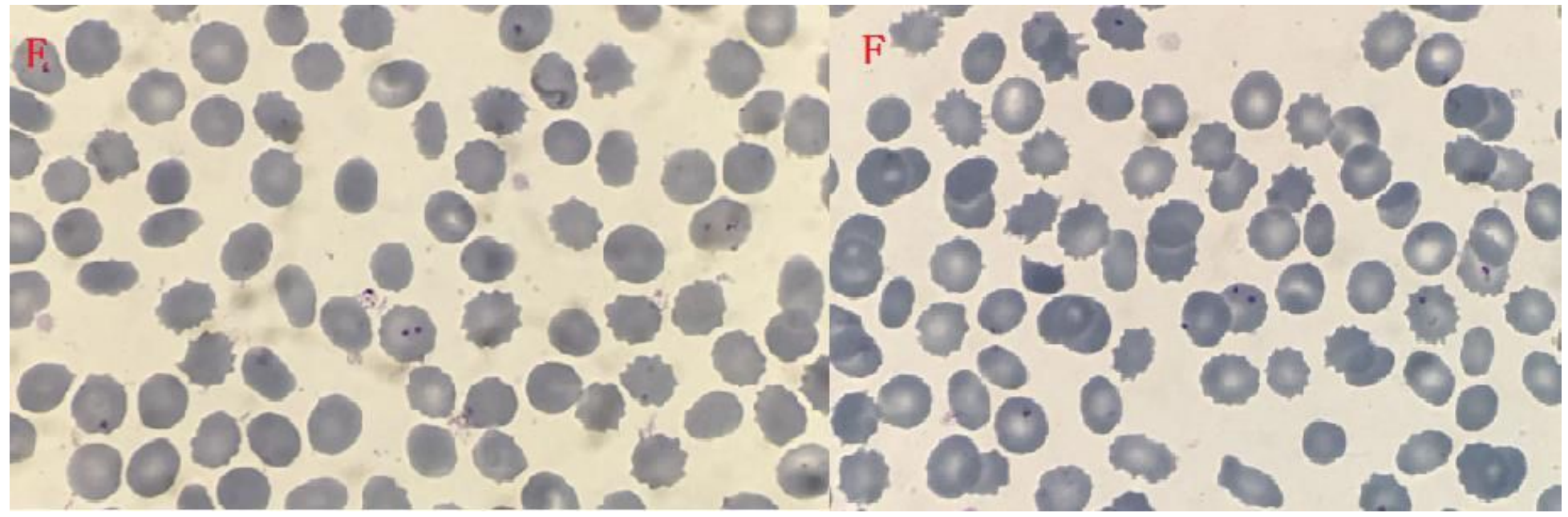

Figure 3 
Plasmodium annulus after treatment (E囚F囚Giemsa stain, $\times 1000)$
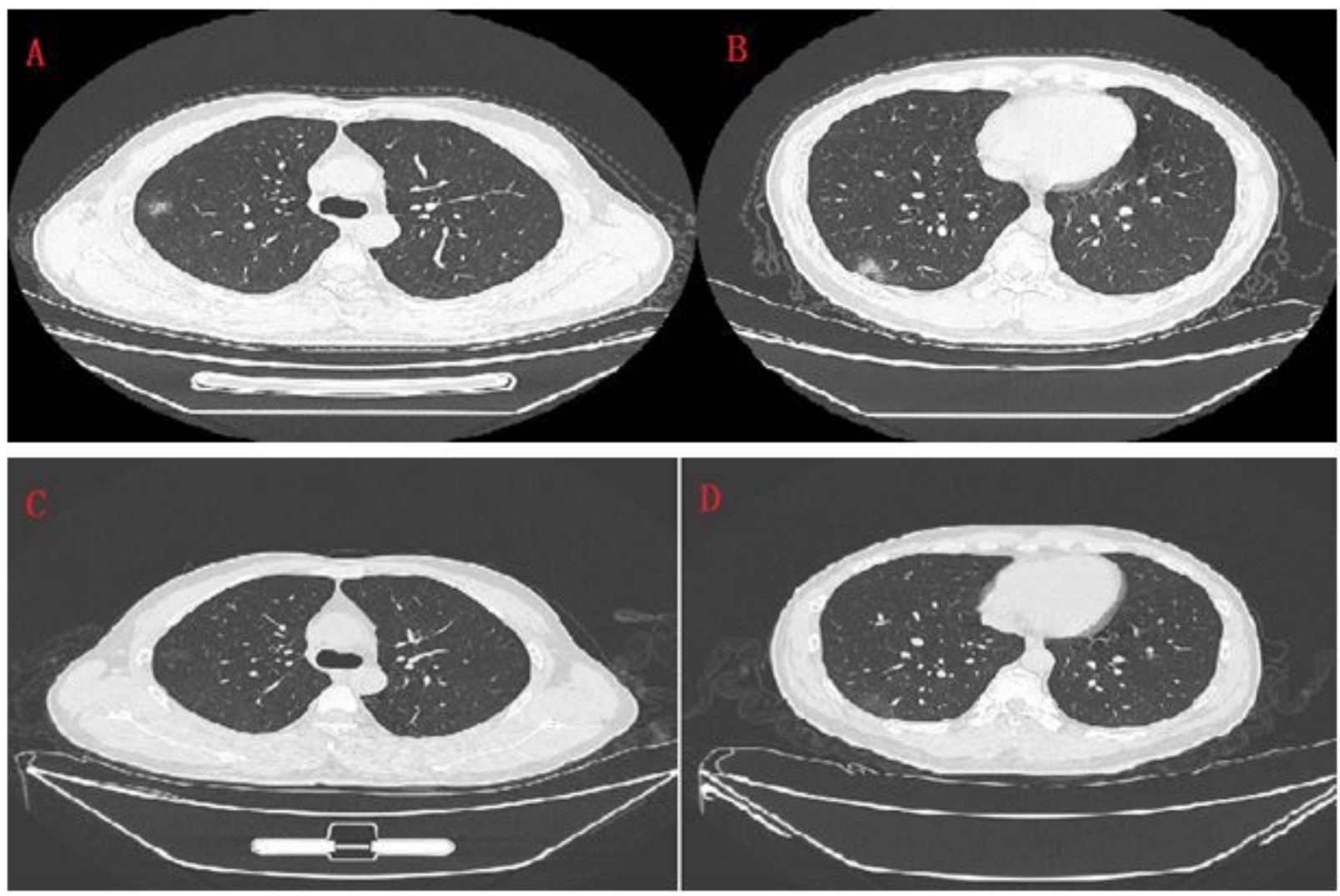

\section{Figure 4}

Comparison of CT imaging results before and after treatment(A and $B$ were the first $C T$ imaging result $\triangle A$ little ground glass density shadow can be seen along the pleural margin of both lungs $₫ C$ and $D$ were the result of CT imaging one week after antiviral therapy $₫ T$ The density of ground glass in the lungs disappeared) 